\title{
The Presence of Ascites Affects the Predictive Value of HVPG on Early Rebleeding in Patients with Cirrhosis
}

\author{
Chuan Liu,, ${ }^{1}$ Ruoyang Shao, ${ }^{2}$ Sining Wang, ${ }^{3}$ Guangchuan Wang, ${ }^{3}$ Lifen Wang, ${ }^{3}$ \\ Mingyan Zhang, ${ }^{3}$ Yanna Liu, ${ }^{4}$ Mingkai Liang, ${ }^{4}$ Xiaoguo Li, ${ }^{4}$ Ning Kang, ${ }^{4}$ Jitao Wang, \\ Dan Xu, ${ }^{4}$ Hua Mao $\mathbb{D}^{1}{ }^{1}$ Chunqing Zhang $\mathbb{D}^{,},{ }^{3}$ and Xiaolong $\mathrm{Qi}^{4}$ \\ ${ }^{1}$ Department of Gastroenterology, Zhujiang Hospital, Southern Medical University, Guangzhou 510000, China \\ ${ }^{2}$ Department of Hematology, Nanfang Hospital, Southern Medical University, Guangzhou 510000, China \\ ${ }^{3}$ Department of Gastroenterology, Shandong Provincial Hospital, Shandong University, Jinan 250000, China \\ ${ }^{4}$ CHESS Center, Institute of Portal Hypertension, The First Hospital of Lanzhou University, Lanzhou 730000, China
}

Correspondence should be addressed to Hua Mao; huam@fimmu.com and Chunqing Zhang; zhangchunqing_sdu@163.com

Received 2 May 2020; Revised 9 August 2020; Accepted 12 November 2020; Published 25 November 2020

Academic Editor: Saurabh Chawla

Copyright (c) 2020 Chuan Liu et al. This is an open access article distributed under the Creative Commons Attribution License, which permits unrestricted use, distribution, and reproduction in any medium, provided the original work is properly cited.

\begin{abstract}
Background and Aims. Gastroesophageal variceal bleeding is a serious complication of portal hypertension in cirrhotic patients and could be predicted by hepatic venous pressure gradient (HVPG). However, whether the presence of ascites affects the prognostic value of HVPG for patients with acute variceal bleeding is still unknown. This retrospective study is aimed at investigating the influence of ascites on predictive performance of HVPG for early rebleeding in cirrhotic patients with acute variceal bleeding. Methods. In this retrospective study, a total of 148 patients with cirrhosis hospitalized for acute variceal bleeding who underwent HVPG measurement and endoscopic variceal ligation (EVL) for the prevention of rebleeding were included. The receiver operating characteristic curve (ROC) and logistical regression method were employed to analyze the predictive performance of HVPG for early rebleeding. The locally weighted scatterplot smoothing approach was adopted to assess the monotonicity between bleeding risk and HVPG. Results. A significantly higher HVPG level was observed in patients with early rebleeding compared to patients without rebleeding in the nonascites cohort. When using HVPG to predict early rebleeding, there was a lower area under curve in the ascites cohort compared to the nonascites cohort. HVPG was recognized as a risk factor for early rebleeding by a logistic regression model only in the nonascites cohort. An overall monotonicity in the trend of change in HVPG and risk for early rebleeding was observed in the nonascites cohort solely. Conclusion. The predictive value of HVPG for early rebleeding in patients with cirrhosis that developed acute variceal bleeding is hindered by the presence of ascites.
\end{abstract}

\section{Introduction}

Gastroesophageal variceal bleeding (GVB) is among the most serious complications of portal hypertension in patients with cirrhosis and even leads to death [1]. Hepatic venous pressure gradient (HVPG) is a potent prognostic factor for patients with cirrhosis [2-4] and has been widely recommended to predict the presence of ascites, hepatic encephalopathy, variceal bleeding and rebleeding, and bleeding-related death [4, 5]. An HVPG higher than $20 \mathrm{mmHg}$ indicates a significantly higher risk of early rebleeding in patients with acute variceal bleeding (AVB) [3, 6-8].
Other than GVB, ascites is also commonly developed in patients with cirrhosis especially those with more advanced disease condition. The enhancing activation of reninangiotensin-aldosterone system (RAAS) as the disease progresses is considered the main pathophysiological process to induce the generation of ascites [9]. Thus, compared to patients without ascites, patients with ascites have a generally worse liver function and more intense hyperdynamic condition that causes instability in hemodynamics [10]. Besides, patients with multiple decompensation events like variceal bleeding combined with ascites, namely, patients experiencing "further decompensation," have worse prognosis than 
those with one decompensation event $[11,12]$. Furthermore, ascites itself as a physical influential factor could also play a disturbing role during HVPG measurement. While it is clear that ascites could influence hemodynamics $[9,10]$, there still lacks evidence to show whether the presence of ascites affects the prognostic value of HVPG in patients with AVB. In this study, we aim to investigate the influence of ascites on the predictive performance of HVPG for early rebleeding in cirrhotic patients with AVB.

\section{Patients and Methods}

2.1. Study Population. In this study, a total of 148 consecutive patients with cirrhosis were retrospectively recruited from Shandong Provincial Hospital between October 2010 and August 2018. The inclusion criteria were as follows: (1) patients hospitalized for AVB with clinically and/or pathologically diagnosed cirrhosis; (2) patients who received octreotide and emergency endoscopic therapy as an initial intervention to stop the acute bleeding and then endoscopic variceal ligation (EVL) (combined with nonselective betablocker (NSBB), or alone when there was an NSBB contraindication) for preventing rebleeding; (3) patients who accepted transjugular HVPG measurement after the emergency endoscopic therapy and within 7 days before and 18 days after the first therapy among the following EVL sequence; and (4) patients who were followed up till the $42^{\text {nd }}$ day or developed rebleeding since accepting EVL. To avoid the influence of EVL on the accuracy of HVPG, patients who accepted HVPG measurement within 48 hours after EVL were excluded [13]. Early rebleeding was defined as rebleeding occurred within 42 days since EVL.

2.2. HVPG Measurement. HVPG measurements were performed using balloon catheters with a pressure transducer at the tip (Edwards Lifesciences, Irvine, Calif) complying with a reported protocol [14]. Before catheterization, a "zero measurement" was performed. The right hepatic vein was chosen for measurements whenever feasible. If stenosis or vein-to-vein shunt in the right hepatic vein was observed, the middle hepatic vein was chosen instead. The free hepatic venous pressure was measured close to the inferior vena cava (1-3 cm, approximately). Then, the balloon was inflated to occlude completely the chosen hepatic vein, and then, the wedged hepatic venous pressure was measured. Dynamic screening of each pressure was continued until the pressure reached a plateau, after which the values were recorded. All measurements were performed in triplicate at least, and the average value was taken as the result. HVPG was determined by subtracting the free hepatic venous pressure from the wedged hepatic venous pressure.

2.3. Statistical Analysis. Continuous variables were shown as the mean and standard deviation (SD) or median and interquartile range (IQR). Categorical variables were shown as the number and frequency (\%). The Mann-Whitney test was used to compare HVPG between nonearly rebleeding and early rebleeding in the ascites and nonascites subgroups. The receiver operating characteristic curve (ROC) was used to evaluate the predictive performance of HVPG for early rebleeding in the nonascites cohort and the ascites cohort, respectively. Univariate and multivariate logistic regression models were employed to calculate odds ratio (OR) and $P$ value of HVPG and other potential risk stratification factors for rebleeding. For a multivariate logistic regression model, platelet (PLT), albumin (ALB), and HVPG were included. The locally weighted scatterplot smoothing (LOWESS) approach was adopted to assess the monotonicity between bleeding risk and HVPG in patients with and without ascites. All levels of significance were set at a two-sided 5\% level. All analyses were performed using SPSS 22.0 IBM (IBM Corp., Armonk, NY) and R 3.5.3 (R Project for Statistical Computing, Vienna, Austria).

\section{Results}

3.1. Patients. A total of 148 patients meeting the inclusion and exclusion criteria were included, of which 106 patients received either propranolol or carvedilol combined with EVL. Patients included were followed up until at least the $42^{\text {nd }}$ day or developed rebleeding since EVL. Early rebleeding occurred in 15 out of 148 patients (10.1\%). Clinical characteristics of the studied cohorts are summarized in Table 1.

\subsection{HVPG Remains Stable in Patients with Ascites Who} Developed Early Rebleeding. During follow-up, 10 out of 79 patients with ascites (ascites cohort) and 5 out of 69 patients without ascites (nonascites cohort) experienced early rebleeding. We compared the HVPG level between patients with and without early rebleeding in both cohorts. In the nonascites cohort, a significantly higher HVPG level was observed in patients experienced early rebleeding compared to those did not $(21.00 \mathrm{mmHg}$ vs. $13.00 \mathrm{mmHg}, P=0.009)$ (Figure 1(a)). However, there was no significant difference in the HVPG level between patients with and without early rebleeding in the ascites cohort (Figure 1(b), median, 17.50 (12.34-21.00) mmHg vs. 14.50 (12.00-18.00) $\mathrm{mmHg}, P=0.207)$.

3.3. Ascites Affects the Predictive Value of HVPG for Early Rebleeding. We used the area under the ROC curve (AUC) to assess whether the presence of ascites affects the predictive value of HVPG on early rebleeding. The ROC curves were plotted for the whole cohort, the ascites cohort, and the nonascites cohort (Figure 2). AUC values of HVPG for predicting early rebleeding showed a tendency to decrease in the three cohorts (AUC: 0.711 (0.570-0.851), 0.852 (0.694-1.000), and 0.624 (0.426-0.822) for whole, nonascites, and ascites cohorts, respectively) (Figure 2).

3.4. The Impact of HVPG on the Risk of Early Rebleeding Is Different in Patients with and without Ascites. To investigate the risk factors for early rebleeding in patients with and without ascites, univariate and multivariate logistic regression analysis were performed. In the nonascites cohort, HVPG was recognized as the only statistically significant risk factor with ORs of $1.350 \quad(P=0.020$, univariate $)$ and 1.350 ( $P=0.029$, multivariate) (Table 2$)$. However, in the ascites cohort, HVPG failed to manifest a significant impact on the 
TABLE 1: Clinical characteristics of the studied patients.

\begin{tabular}{|c|c|c|c|c|}
\hline Variables & Patients $(n=148)$ & Ascites group $(n=79)$ & Nonascites group $(n=69)$ & $P$ \\
\hline Age $(y)$, median (IQR) & $51.5(15.75)$ & $53.0(16.00)$ & $50.0(13.50)$ & 0.071 \\
\hline Gender, $n(\%)$ & & & & 0.607 \\
\hline Male & $46(31.1)$ & $53(67.1)$ & $20(29.0)$ & \\
\hline Female & $102(68.9)$ & $26(32.9)$ & $49(71.0)$ & \\
\hline AST (IU/L), median (IQR) & $33.5(20.5)$ & $34.0(19.0)$ & $33.0(24.0)$ & 0.745 \\
\hline ALT (IU/L), median (IQR) & $25.0(15.8)$ & $25.0(19.0)$ & $25.0(14.0)$ & 0.917 \\
\hline $\operatorname{PLT}\left(10^{9} / \mathrm{L}\right)$, median (IQR) & $71.5(72.5)$ & $66.0(71.0)$ & $83.0(78.0)$ & 0.118 \\
\hline TBIL ( $\mu \mathrm{mol} / \mathrm{L})$, median (IQR) & $19.9(10.7)$ & $20.5(12.1)$ & $18.9(9.85)$ & 0.138 \\
\hline ALB (g/L), median (IQR) & $33.4(7.4)$ & $31.2(7.7)$ & $34.7(7.4)$ & $<0.001$ \\
\hline INR, median (IQR) & $1.21(0.25)$ & $1.24(0.25)$ & $1.20(0.16)$ & 0.019 \\
\hline Accepting NSBB, $n(\%)$ & $106(71.6)$ & $48(60.76)$ & $58(84.06)$ & 0.002 \\
\hline Ascites, $n(\%)$ & $79(53.4)$ & NA & NA & NA \\
\hline Early rebleeding, $n(\%)$ & $15(10.1)$ & $10(12.66)$ & $5(7.25)$ & 0.414 \\
\hline HVPG (mmHg), mean (SD) & $15.0(4.66)$ & $15.46(4.52)$ & $14.44(4.79)$ & 0.158 \\
\hline Child-Pugh class, $n(\%)$ & & & & $<0.001$ \\
\hline Child A & $62(41.9)$ & $14(17.72)$ & $48(69.57)$ & \\
\hline Child B & $73(49.4)$ & $53(67.09)$ & $20(28.99)$ & \\
\hline Child C & $13(8.8)$ & $12(15.19)$ & $1(1.45)$ & \\
\hline Etiology, $n(\%)$ & & & & 0.165 \\
\hline Viral & $87(58.7)$ & $53(67.09)$ & $34(49.28)$ & \\
\hline Alcoholic & $16(10.8)$ & $8(10.13)$ & $8(11.59)$ & \\
\hline Autoimmunogenic & $10(6.7)$ & $5(6.33)$ & $5(7.25)$ & \\
\hline Cholestatic & $5(3.4)$ & $1(1.27)$ & $4(5.80)$ & \\
\hline Other & $30(20.3)$ & $12(15.19)$ & $18(26.09)$ & \\
\hline
\end{tabular}

AST: aspartate aminotransferase; ALT: alanine aminotransferase; PLT: platelets; TBIL: total bilirubin; INR: international normalized; ALB: albumin; NSBB: nonselective beta-blocker; MELD: Model of End-stage Liver Disease; HVPG: hepatic venous pressure gradient; y: years; IQR: interquartile range.

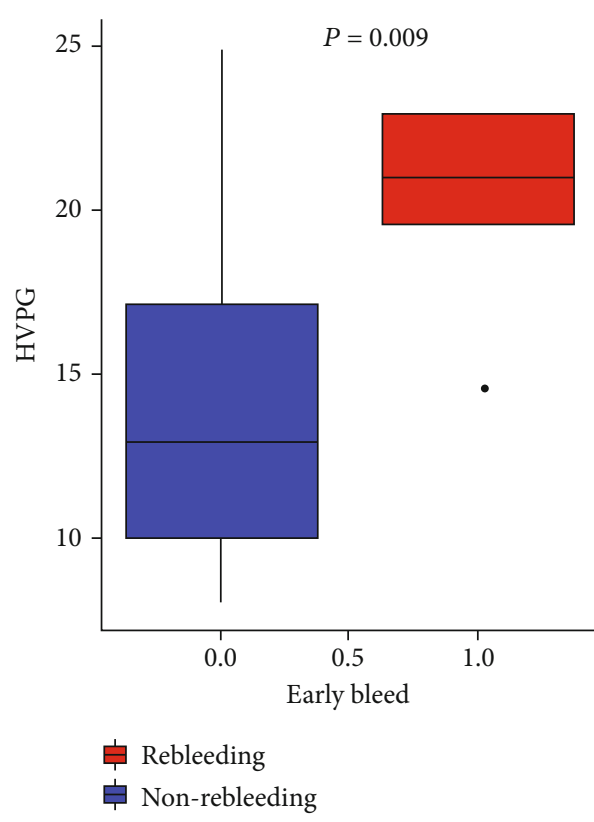

(a)

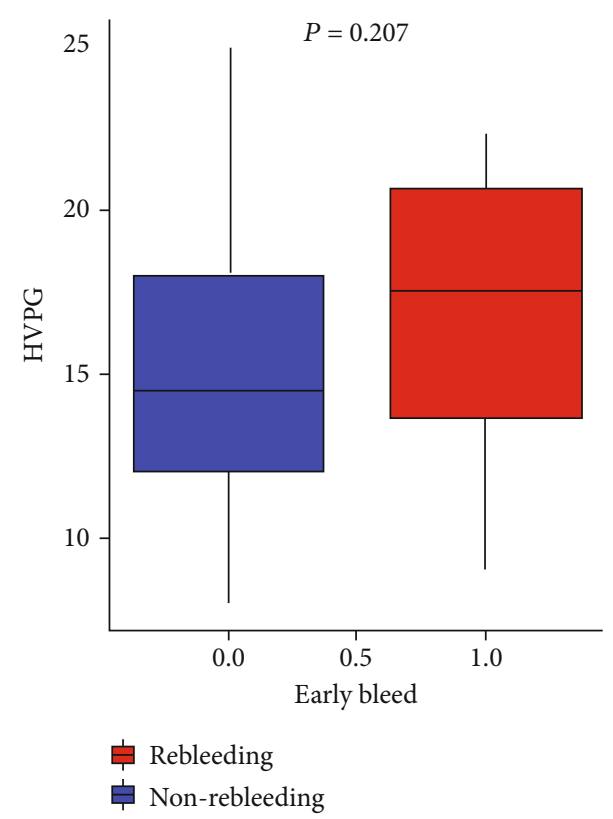

(b)

FIGURE 1: Comparisons of HVPG in patients with and without early rebleeding in (a) the nonascites cohort and (b) the ascites cohort. HVPG: hepatic venous pressure gradient. 


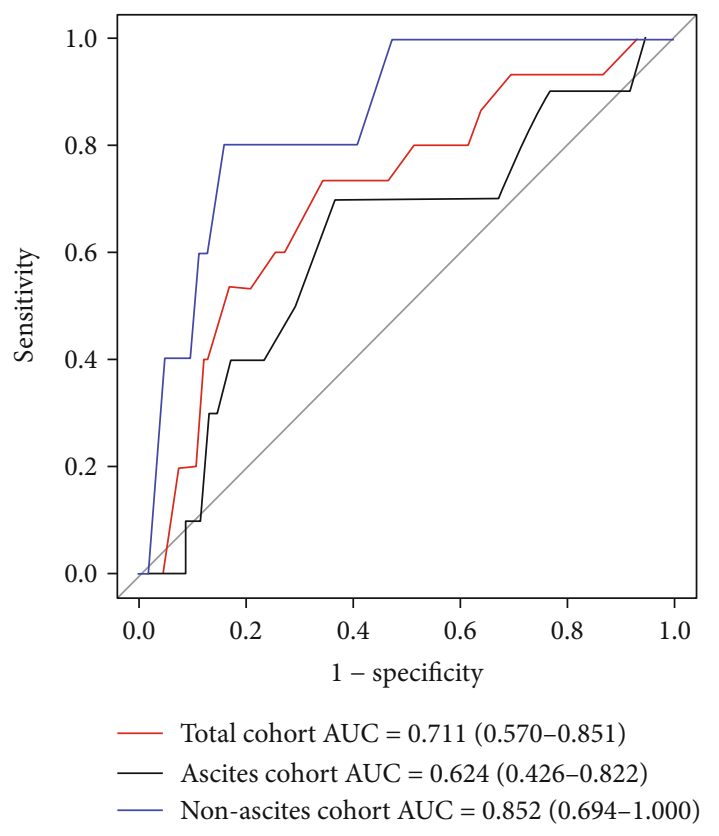

FIGURE 2: ROC curve of HVPG for predicting early rebleeding in the total cohort, the ascites cohort, and the nonascites cohort. AUC: area under the ROC curve.

risk of early rebleeding with ORs of $1.089(P=0.253$, univariate) and $1.073(P=0.380$, multivariate) (Table 3$)$.

It is generally believed that the higher the HVPG of cirrhotic patients, the higher risk for rebleeding they suffer. So we believe that if the HVPG level is a risk factor of early rebleeding in a certain population, there should be an overall monotonicity in the trend of change in HVPG and the risk for early rebleeding. Therefore, we employed the LOWESS approach to generate a fitting curve that reflected the overall trend of change in HVPG and risk for early rebleeding in the ascites cohort and the nonascites cohort, respectively, in order to assess their monotonicity. As shown in Figure 3, an overall monotonicity was observed in the nonascites cohort but not in the ascites cohort.

\section{Discussion}

HVPG could filter the influence of the central venous system and abdominal pressure and is widely accepted as an accurate index for assessing portal hypertension [15-17]. It has been proved to be a potent and versatile prognostic factor in cirrhotic portal hypertension. HVPG $\geq 10 \mathrm{mmHg}$ is regarded as the threshold for the occurrence of decompensation and is thus called clinically significant portal hypertension. Patients with clinically significant portal hypertension face significantly higher risks of developing varices, bleeding, other decompensation events, and hepatocellular carcinoma $[2,18,19]$. Patients with an HVPG $\geq 16 \mathrm{mmHg}$ suffer from higher mortality [20-22] and bleeding risk [7, 23]. An HVPG above $20 \mathrm{mmHg}$ is strongly predictive of failure to control bleeding, early rebleeding, and hemorrhage-related death $[3,24]$.

It is intuitive and generally successful to stratify bleeding risk using the stable portal pressure reflector, HVPG, based on the direct correlation between the elevation of portal pressure and risk of varices bleeding. However, there still exist confounding factors affecting either the accuracy of HVPG measurement or its capability to indicate the actual bleeding risk in patients with cirrhosis, especially those with more complex disease conditions, like patients with ascites.

In patients with cirrhosis, the presence of ascites is the consequence of the activation of RAAS initiated by portal hypertension. Approximately $60 \%$ of cirrhotic patients develop ascites in 10 years since diagnosis [25], and ascites is the first decompensation event in most patients [26, 27].

In studies that support the role for an HVPG higher than $20 \mathrm{mmHg}$ to indicate a higher risk of treatment failure or early rebleeding, none of them performed subgroup analysis for patients with and without ascites $[3,6-8,28]$. However, as stated above, patients with ascites have generally more advanced disease condition and poorer liver function and are therefore more easily to develop endothelial dysfunction [29]. Under these circumstances, HVPG could not accurately reflect the portal pressure for it actually represents the pressure of the hepatic sinusoid. Besides, patients with ascites are in a more intense hyperdynamic state and with more unstable hemodynamics $[10,30]$. These patients, even with relatively low HVPG, may suffer from higher risks of rapid increment of HVPG and exacerbation of disease that results in worse clinical outcomes, compared to patients with similar HVPG but without ascites. A significantly higher mortality was observed in patients with ascites compared to patients without any decompensation events, and the result was also similar when comparing patients with ascites and experienced bleeding to those who experienced bleeding but without other decompensation events [11, 12]. Also, although the elevation of portal pressure is considered the dominant factor of bleeding, the more complex condition in patients with ascites inevitably adds more influential factors and thus hinders the predictive performance of the single predictor, HVPG. Additionally, although HVPG could filter the influence of the central venous system and abdominal pressure theoretically, the measurement error introduced by respiratory cycle cannot be eliminated [31].

One possible solution to improve the early rebleedingpredictive performance is to combine HVPG with other clinical indicators to develop an extended predictive model. In a meta-analysis that included 118 studies, Child-Pugh, encephalopathy, hepatocellular carcinoma, bleeding, creatinine, prothrombin time, albumin, azotemia, ascites, and bilirubin were shown to be frequently used statistically significant prognostic parameters in patients with decompensated cirrhosis [32]. By introducing other clinical indicators, a model that covers different factors that influence clinical outcome from different aspects could be developed. A multiple factor model may be able to reflect the disease condition of patients in a more comprehensive manner, resulting in possible improvement in predictive performance. However, the more indicators included in a model, the less easy-touse the model will be. Another possible attempt is to track the change of HVPG after acute bleeding. As reported by Ready et al., acute bleeding patients who did not develop early rebleeding showed an overall decreasing trend of HVPG after 
TABLE 2: Univariate and multivariate logistic regression analysis in the nonascites cohort.

\begin{tabular}{lccc}
\hline Variable & OR & Univariate & OR \\
\hline Child-Pugh score & $0.543(0.061-4.807)$ & 0.583 & $P$ valtivariate \\
HVPG & $1.350(1.049-1.737)$ & 0.020 & $1.350(1.032-1.765)$ \\
AST & $0.988(0.932-1.047)$ & 0.681 & 0.029 \\
ALT & $0.9996(0.954-1.040)$ & 0.863 & \\
ALB & $1.066(0.889-1.278)$ & 0.490 & \\
TBIL & $0.998(0.979-1.018)$ & 0.850 & \\
PLT & $0.997(0.983-1.010)$ & 0.614 & \\
INR & $6.989(0.061-804.738)$ & 0.422 & 0.996 \\
\hline
\end{tabular}

HR: hazard ratio; HVPG: hepatic venous pressure gradient; AST: aspartate aminotransferase; ALT: alanine aminotransferase; ALB: albumin; TBIL: total bilirubin; PLT: platelets; INR: international normalized ratio.

TABLE 3: Univariate and multivariate logistic regression analysis in the ascites cohort.

\begin{tabular}{lccc}
\hline Variable & OR & Univariate & OR \\
\hline Child-Pugh score & $3.278(0.952-11.289)$ & 0.060 & $P$ value \\
HVPG & $1.089(0.941-1.261)$ & 0.253 & $1.073(0.917-1.255)$ \\
AST & $0.980(0.942-1.019)$ & 0.310 & 0.380 \\
ALT & $0.969(0.916-1.025)$ & 0.267 & \\
ALB & $0.965(0.864-1.078)$ & 0.531 & \\
TBIL & $1.026(0.993-1.061)$ & 0.125 & \\
PLT & $0.980(0.957-1.004)$ & 0.096 & $14.364(0.825-250.056)$ \\
INR & $17.052(1.014-286.888)$ & 0.049 & 0.068 \\
\hline
\end{tabular}

HR: hazard ratio; HVPG: hepatic venous pressure gradient; AST: aspartate aminotransferase; ALT: alanine aminotransferase; ALB: albumin; TBIL: total bilirubin; PLT: platelets; INR: international normalized ratio.

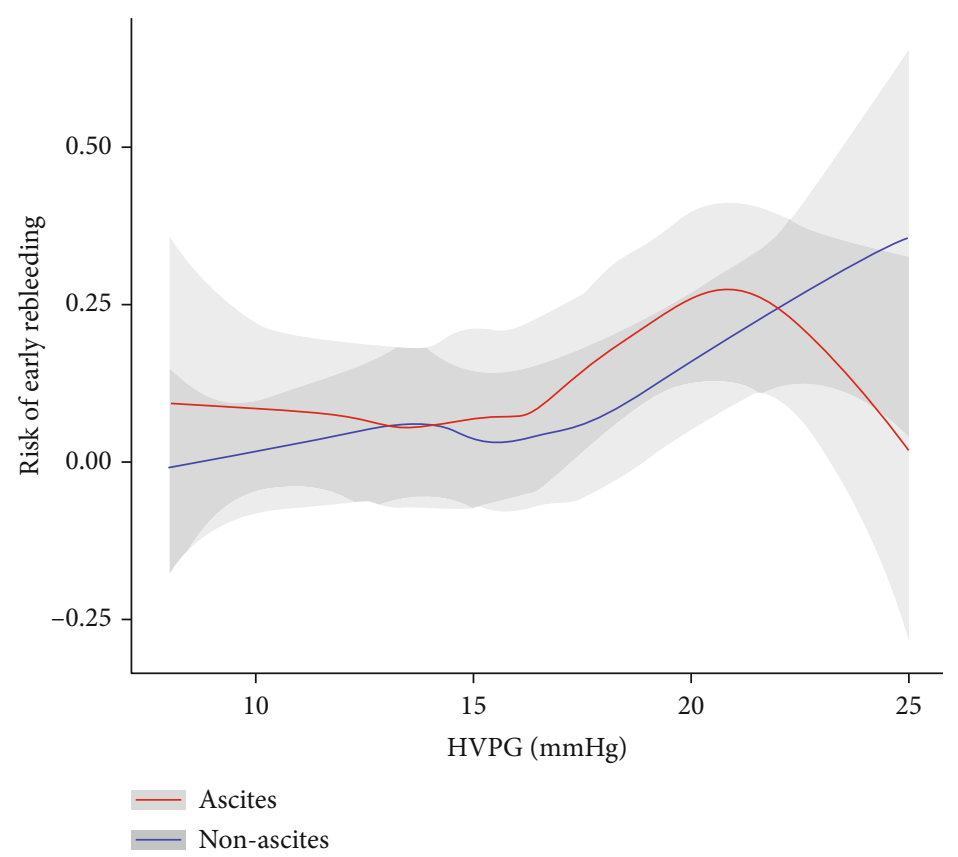

FIGURE 3: LOWESS curve for assessing the trend of risk for early rebleeding on HVPG in the ascites cohort and nonascites cohort. 
the acute phase [28]. Overcoming the invasiveness and high cost of extra HVPG measurements, the emerging techniques for noninvasive prediction of portal pressure have achieved high accuracy using routine clinical data [1, 33-35]. These serum- or imaging-based methods may provide additional data that benefit our decision. Nevertheless, the sensitivity of these methods to the short-term change of portal pressure remains to be tested before being applied for dynamic monitoring.

Our study for the first time investigated the influence of ascites on the predictive value of HVPG for early rebleeding in cirrhotic patients with AVB. Yet, there are also several limitations. First, this study is a retrospective study including cases from a single center, which may be a possible source of bias. Second, subgroup analysis was not performed for patients with ascites of different intensities due to lack of original data. Third, not all the patients included received NSBB, and this heterogeneity may also be a source of bias. Fourth, patients were followed up for only 42 days, so no data on other events could be provided.

In summary, we found that patients with early rebleeding have a higher HVPG than those who did not in the nonascites cohort, but not in the ascites cohort. When using HVPG to predict early rebleeding, the AUC in the ascites cohort was significantly lower comparing to the nonascites cohort and the whole cohort. HVPG was recognized as a risk factor for early rebleeding in the nonascites cohort but not in the ascites cohort. An overall monotonicity in the trend of change in HVPG and risk for early rebleeding was observed in the nonascites cohort only using the LOWESS approach. Taking together, these findings suggested that the predictive value of HVPG for early rebleeding in patients with cirrhosis that developed AVB is hindered by the presence of ascites.

\section{Data Availability}

The data that support the findings of this study are available from the corresponding author upon reasonable request.

\section{Conflicts of Interest}

The authors declare no conflict of interest.

\section{Authors' Contributions}

Hua Mao, Chunqing Zhang, and Xiaolong Qi are responsible for the study concept and design. Sining Wang, Guangchuan Wang, Lifen Wang, and Mingyan Zhang acquired the data. Chuan Liu, Ruoyang Shao, Yanna Liu, Mingkai Liang, Xiaoguo Li, Ning Kang, Jitao Wang, and Dan Xu performed the analysis and interpretation of data. Ruoyang Shao and Sining Wang drafted the manuscript. Chunqing Zhang, Hua Mao, and Xiaolong Qi are assigned to the critical revision of the manuscript. Chuan Liu, Ruoyang Shao, and Sining Wang contributed equally to this work.

\section{Acknowledgments}

This study received no commercial support and has been supported by Guangdong Science Fund for Distinguished
Young Scholars (2018B030306019) and Guangzhou Industry-Academia-Research Collaborative Innovation Major Project (201704020015).

\section{References}

[1] X. Qi, A. Berzigotti, A. Cardenas, and S. K. Sarin, "Emerging non-invasive approaches for diagnosis and monitoring of portal hypertension," The Lancet Gastroenterology \& Hepatology, vol. 3, no. 10, pp. 708-719, 2018.

[2] C. Ripoll, R. Groszmann, G. Garcia-Tsao et al., "Hepatic venous pressure gradient predicts clinical decompensation in patients with compensated cirrhosis," Gastroenterology, vol. 133, no. 2, pp. 481-488, 2007.

[3] E. Moitinho, A. Escorsell, J. C. Bandi et al., "Prognostic value of early measurements of portal pressure in acute variceal bleeding," Gastroenterology, vol. 117, no. 3, pp. 626-631, 1999.

[4] G. Garcia-Tsao, J. G. Abraldes, A. Berzigotti, and J. Bosch, "Portal hypertensive bleeding in cirrhosis: risk stratification, diagnosis, and management: 2016 practice guidance by the American Association for the study of liver diseases," Hepatology, vol. 65, no. 1, pp. 310-335, 2016.

[5] R. Franchis and Baveno VI Faculty, "Expanding consensus in portal hypertension report of the Baveno VI Consensus Workshop: stratifying risk and individualizing care for portal hypertension," Journal of Hepatology, vol. 63, no. 3, pp. 743-752, 2015.

[6] J. G. Abraldes, C. Villanueva, R. Bañares et al., "Hepatic venous pressure gradient and prognosis in patients with acute variceal bleeding treated with pharmacologic and endoscopic therapy," Journal of Hepatology, vol. 48, no. 2, pp. 229-236, 2008.

[7] G.-Q. Li, B. Yang, J. Liu et al., "Hepatic venous pressure gradient is a useful predictor in guiding treatment on prevention of variceal rebleeding in cirrhosis," International Journal of Clinical and Experimental Medicine, vol. 8, no. 10, pp. 1970919716, 2015.

[8] J. R. Zhao, G. C. Wang, J. H. Hu, and C. Q. Zhang, "Risk factors for early rebleeding and mortality in acute variceal hemorrhage," World Journal of Gastroenterology, vol. 20, no. 47, pp. 17941-17948, 2014.

[9] M. Bernardi, R. Moreau, P. Angeli, B. Schnabl, and V. Arroyo, "Mechanisms of decompensation and organ failure in cirrhosis: from peripheral arterial vasodilation to systemic inflammation hypothesis," Journal of Hepatology, vol. 63, no. 5, pp. 1272-1284, 2015.

[10] L. Blendis and F. Wong, "The hyperdynamic circulation in cirrhosis : an overview.," Pharmacology \& Therapeutics, vol. 89, 2001.

[11] G. D'Amico, A. Morabito, M. D'Amico et al., "Clinical states of cirrhosis and competing risks," Journal of Hepatology, vol. 68, no. 3, pp. 563-576, 2018.

[12] G. D’Amico, A. Morabito, M. D’Amico et al., "New concepts on the clinical course and stratification of compensated and decompensated cirrhosis," Hepatology International, vol. 12, no. S1, pp. 34-43, 2018.

[13] A. Avgerinos, A. Armonis, G. Stefanidis et al., "Sustained rise of portal pressure after sclerotherapy, but not band ligation, in acute variceal bleeding in cirrhosis," Hepatology, vol. 39, no. 6, pp. 1623-1630, 2004. 
[14] R. J. Groszmann and S. Wongcharatrawee, "The hepatic venous pressure gradient: anything worth doing should be done right," Hepatology, vol. 39, no. 2, pp. 280-283, 2004.

[15] J. D. Vorobioff, "Hepatic venous pressure in practice," Journal of Clinical Gastroenterology, vol. 41, Supplement 3, pp. S336S343, 2007.

[16] A. Kumar, P. Sharma, and S. K. Sarin, "Hepatic venous pressure gradient measurement: time to learn!," Indian Journal of Gastroenterology, vol. 27, pp. 74-80, 2008.

[17] B. Procopet and C. Bureau, "Invasive and non-invasive diagnosis of portal hypertension in cirrhosis," Current Hepatology Reports, vol. 13, no. 3, pp. 180-188, 2014.

[18] R. J. Groszmann, G. Garcia-Tsao, J. Bosch et al., "Beta-blockers to prevent gastroesophageal varices in patients with cirrhosis," The New England Journal of Medicine, vol. 353, no. 21, pp. 2254-2261, 2005.

[19] C. Ripoll, R. J. Groszmann, G. Garcia-Tsao et al., "Hepatic venous pressure gradient predicts development of hepatocellular carcinoma independently of severity of cirrhosis," Journal of Hepatology, vol. 50, no. 5, pp. 923-928, 2009.

[20] G. Garcia-Tsao, R. J. Groszmann, R. L. Fisher, H. O. Conn, C. E. Atterbury, and M. Glickman, "Portal pressure, presence of gastroesophageal varices and variceal bleeding," Hepatology, vol. 5, no. 3, pp. 419-424, 1985.

[21] A. Berzigotti, V. Rossi, C. Tiani et al., "Prognostic value of a single HVPG measurement and Doppler-ultrasound evaluation in patients with cirrhosis and portal hypertension," Journal of Gastroenterology, vol. 46, no. 5, pp. 687-695, 2011.

[22] G. Silva-Junior, A. Baiges, F. Turon et al., "The prognostic value of hepatic venous pressure gradient in patients with cirrhosis is highly dependent on the accuracy of the technique," Hepatology, vol. 62, no. 5, pp. 1584-1592, 2015.

[23] C. Merkel, M. Bolognesi, S. Bellon et al., "Prognostic usefulness of hepatic vein catheterization in patients with cirrhosis and esophageal varices," Gastroenterology, vol. 102, no. 3, pp. 973-979, 1992.

[24] A. Monescillo, F. Martinez-Lagares, L. Ruiz-del-Arbol et al., "Influence of portal hypertension and its early decompression by TIPS placement on the outcome of variceal bleeding," Hepatology, vol. 40, no. 4, pp. 793-801, 2004.

[25] E. Pose and A. Cardenas, "Translating our current understanding of ascites management into new therapies for patients with cirrhosis and fluid retention," Digestive Diseases, vol. 35, no. 4, pp. 402-410, 2017.

[26] R. Jalan, F. Saliba, M. Pavesi et al., "Development and validation of a prognostic score to predict mortality in patients with acute-on-chronic liver failure," Journal of Hepatology, vol. 61, no. 5, pp. 1038-1047, 2014.

[27] G. D'Amico, L. Pasta, A. Morabito et al., "Competing risks and prognostic stages of cirrhosis: a 25-year inception cohort study of 494 patients," Alimentary Pharmacology \& Therapeutics, vol. 39, no. 10, pp. 1180-1193, 2014.

[28] J. B. Ready, A. D. Robertson, J. S. Goff, and W. G. Rector Jr., "Assessment of the risk of bleeding from esophageal varices by continuous monitoring of portal pressure," Gastroenterology, vol. 100, no. 5, pp. 1403-1410, 1991.

[29] B. Shenoda and J. Boselli, "Vascular syndromes in liver cirrhosis," Clinical Journal of Gastroenterology, vol. 12, no. 5, pp. 387-397, 2019.

[30] C. Villanueva, A. Albillos, J. Genescà et al., "Development of hyperdynamic circulation and response to $\beta$-blockers in com- pensated cirrhosis with portal hypertension," Hepatology, vol. 63, no. 1, pp. 197-206, 2016.

[31] E. Reverter, A. Blasi, J. G. Abraldes et al., "Impact of deep sedation on the accuracy of hepatic and portal venous pressure measurements in patients with cirrhosis," Liver International, vol. 34 , no. 1 , pp. $16-25,2014$

[32] G. D’Amico, G. Garcia-Tsao, and L. Pagliaro, "Natural history and prognostic indicators of survival in cirrhosis: a systematic review of 118 studies," Journal of Hepatology, vol. 44, no. 1, pp. 217-231, 2006.

[33] J. Dong and X. Qi, "Liver imaging in precision medicine," eBioMedicine, vol. 32, pp. 1-2, 2018.

[34] F. Liu, Z. Ning, Y. Liu et al., "Development and validation of a radiomics signature for clinically significant portal hypertension in cirrhosis (CHESS1701): a prospective multicenter study," eBioMedicine, vol. 36, pp. 151-158, 2018.

[35] X. Qi, W. An, F. Liu et al., "Virtual hepatic venous pressure gradient with CT angiography (chess 1601): a prospective multicenter study for the noninvasive diagnosis of portal hypertension," Radiology, vol. 290, no. 2, pp. 370-377, 2019. 